Exact solution (within a triple-zeta, double polarization basis set) of the electronic Schrödinger equation for water

Garnet Kin-Lic ChanMartin Head-Gordon

Citation: 118, (2003); doi: 10.1063/1.1574318

View online: http://dx.doi.org/10.1063/1.1574318

View Table of Contents: http://aip.scitation.org/toc/jcp/118/19

Published by the American Institute of Physics 


\title{
Exact solution (within a triple-zeta, double polarization basis set) of the electronic Schrödinger equation for water
}

\author{
Garnet Kin-Lic Chan \\ Department of Chemistry, University of Cambridge, United Kingdom CB2 1EW \\ Martin Head-Gordon \\ Department of Chemistry, University of California, Berkeley, California 94070
}

(Received 4 March 2003; accepted 21 March 2003)

\begin{abstract}
Using a newly developed density matrix renormalization group algorithm, we have computed exact solutions of the Schrödinger equation for water at two geometries in a basis of 41 orbitals. Calculations of this size cannot be carried out using any other method. We compare our results with high-order coupled cluster and configuration interaction calculations. (C) 2003 American Institute of Physics. [DOI: 10.1063/1.1574318]
\end{abstract}

Exact solutions of the Schrödinger equation within a given one-particle basis set, lie at the heart of quantum chemistry. Even when the one-particle basis is not very large, such calculations provide invaluable benchmarks by which the accuracy of more approximate methods may be judged.

Using the full configuration interaction method (FCI), advances by Handy and Knowles, ${ }^{1-3}$ Olsen et al., ${ }^{4,5}$ Harrison and Zarrabian, ${ }^{6}$ and Bendazzoli and Evangelisti, ${ }^{7}$ to name but a few, together with rapid improvements in computer technology, now allow calculations involving up to $9 \times 10^{9}$ Slater determinants. The largest such calculation, by Rossi et al. ${ }^{8}$ has been on the $\mathrm{N}_{2}$ molecule, involving 10 electrons and 34 orbitals in $D_{2 h}$ symmetry. However, notwithstanding these accomplishments, the exponential cost of the full configuration interaction algorithm as a function of problem size severely limits the choice of problems that can be studied to high accuracy.

We have been working on an alternative route to exact solutions of the Schrödinger equation, via the density matrix renormalization group (DMRG) algorithm. The DMRG, based on early work by Wilson, ${ }^{9}$ was invented by White ${ }^{10,11}$ in the field of model Hamiltonians (e.g., 1D Hubbard models) in the early 1990s, and first applied to molecular Hamiltonians (of the PPP type) by Fano, Ortolani, and Ziosi. ${ }^{12}$ This was followed by the first DMRG calculation with the full electronic Hamiltonian by White and Martin in $1999 .{ }^{13}$ In the last few years, this method has been actively developed in a number of works, including those of Daul et al., ${ }^{14}$ Mitrushenkov et al., ${ }^{15}$ and Chan and Head-Gordon. ${ }^{16}$

The DMRG differs from usual quantum chemistry methods, in that it avoids the expansion of the wave function in a basis of Slater determinants. Instead, general many-body basis states, which would in principle themselves have long and complicated Slater determinant expansions, are used to expand the wave function. One avoids the need to know the explicit form of such states, by storing matrix representations of all the operators necessary to solve the Schrödinger equation. Then, starting from an "initial guess" Slater determinant expansion for the wave function, one iteratively rotates the relevant operator matrices into a complicated many-body basis, chosen to be eigenvectors of a many-body density matrix. These states are, in a certain well defined sense, ${ }^{10}$ an optimal basis in which to expand the wave function. Each rotation is accompanied by a variational truncation of the many-body basis, so that only a fixed number of states need be considered at any one time.

Because the many-body expansion states are chosen in an optimal way, it is possible, using a small number of expansion states, to achieve a very high accuracy in the energy. The convergence of the energy with the number of expansion states is also very fast, indeed superpolynomial. ${ }^{17}$ With certain Hamiltonians, such as short-ranged 1D Hamiltonians, the number of states needed to achieve a given accuracy in the energy does not increase with the system size. All these characteristics make the DMRG a particularly promising algorithm for performing systematic high accuracy calculations.

In this report, we use the DMRG to exactly solve the Schrödinger equation for the water molecule within a basis of 41 orbitals. We mean exact in the numerical sense, where we compute the energy to an accuracy of one hundredth of a milliHartree $\left(0.01 \mathrm{mE}_{h}\right)$, which is the typical accuracy to which FCI benchmarks are converged. We have chosen the water molecule because of its long and distinguished history as the molecule of choice for benchmark calculations. In 1981, Saxe, Schaefer, and Handy ${ }^{18}$ performed the first largescale FCI for the water molecule in a double-zeta (DZ) basis of 14 functions, which involved $10^{6} C_{2 v}$ determinants. In 1986, Bauschlicher and Taylor ${ }^{19}$ extended these results to a frozen-core double-zeta, polarization (DZP) calculation, involving $10^{7}$ determinants. A decade later, Olsen et al. ${ }^{20}$ carried out FCI in a DZP basis of 26 functions correlating all ten electrons, which corresponded to a total determinantal space of $5 \times 10^{8} C_{2 v}$ determinants. In our calculations, we take the next step, correlating all ten electrons using a triple-zeta, double polarization (TZ2P) quality basis. We have chosen the ANO basis of Widmark, Malmqvist, and Roos ${ }^{21}$ which is a balanced basis for properties and energies, consisting of $4 s 3 p 2 d$ functions on $\mathrm{O}$ and $3 s 2 p$ functions on $\mathrm{H}$ yielding 41 basis functions in total. This may be obtained from the 
Extensible Computational Chemistry Environment Basis Set Database under the name "Roos Augmented Double Zeta ANO." The corresponding determinantal space in $C_{1}$ symmetry (which is the symmetry used in our code and calculations; we have neglected symmetry for simplicity) is 5.6 $\times 10^{11}$ determinants. With full point group symmetry, this is reduced to $1.4 \times 10^{11} C_{2 v}$ determinants. Either size of space, however, is currently out of reach of any traditional FCI algorithm.

In addition to calculations at the equilibrium geometry $\left(r_{e}\right)$, we have also considered a stretched geometry where the $\mathrm{OH}$ bond is stretched to 1.5 times its equilibrium length $\left(1.5 r_{e}\right)$. These geometries correspond to positions (in Angstroms) of $\mathrm{O}(0,0,0), \mathrm{H}( \pm 0.790689766,0,0.612217330)$, and $\mathrm{O}(0,0,0), \mathrm{H}( \pm 1.186034914,0,0.918326260)$, respectively.

In our DMRG algorithm, the orbitals are mapped onto a lattice and divided into four blocks, two large blocks of variable size $S$ and $E$, and two small blocks of constant size $B_{S}$, $B_{E}$ in between. At each step of a DMRG sweep, the size of the system block $S$ is increased by adding more orbitals, while the size of the environment $E$ is decreased. A complete sweep consists of repeatedly adding spin-orbitals to the system (blocking), until the system spans all the orbitals. After a sweep is completed in one direction, the roles of system and environment are reversed, and the sweep is carried out in the opposite direction; each sweep successively improves the description of the system and environment blocks and the process may be iterated to convergence. The system block spans a many-body space $\{S\}$ before blocking; after blocking, the enlarged system block spans the enlarged space $\{S\} \otimes\left\{B_{S}\right\}$. Therefore the many body-states of the system must be truncated, otherwise further blockings will lead to an exponential explosion in the number of states. The optimal choice of system states are the eigenvectors of largest weight (eigenvalue) of the many-body reduced density matrix of the system. To obtain this representation, we determine the groundstate wave function $|\Psi\rangle$ in the product space of the (enlarged) system and environment blocks

$$
|\Psi\rangle=\sum_{S E} c_{S E}|S\rangle|E\rangle
$$

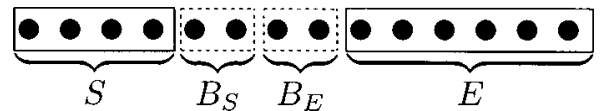

FIG. 1. A standard block configuration. Each - represents a spin-orbital. $B_{S}$ is to be blocked with $S$, and $B_{E}$ with $E$.

and construct the reduced density matrix $\Gamma$ of the system by tracing out the environment states

$$
\Gamma_{S S^{\prime}}=\operatorname{Tr}_{E} c_{S E^{2}} c_{S^{\prime} E}|S\rangle\left\langle S^{\prime}\right| \text {. }
$$

Diagonalization of $\Gamma$ is then followed by the rotation of all the necessary operators into the new eigenvector basis.

A DMRG sweep is specified by the number of spinorbitals in $B_{S}, B_{E}$ at each step, and the number of manybody states $M$ retained at each truncation to span the system block, together with the orbitals themselves. In our sweeps, we use the block configuration shown in Fig. 1, where at each blocking step we add two spin-orbitals.

For a finite number of states $M$ that does not completely span the FCI space, the DMRG is not invariant under unitary transformation of the orbitals (although as $M$ increases, and the calculation becomes exact, invariance is recovered); furthermore, the order in which the orbitals are mapped onto the lattice influences the convergence of the procedure. ${ }^{16}$ In these calculations, we used restricted canonical HartreeFock orbitals at the equilibrium geometry, and unrestricted canonical Hartree-Fock orbitals at the stretched geometry. As discussed in our earlier work ${ }^{16}$ a good criterion to order the orbitals is provided by minimizing the one-body matrix elements between distant orbitals. We have used this criterion to yield the orbital orderings in this work, listed in Table I. We observe that the algorithm naturally produces clusters of virtual orbitals that surround orbitals occupied in the Hartree-Fock reference.

We first performed DMRG sweeps retaining only a small number of states $M$ and then progressively increased $M$. For each of these small $M$ values $(200,350,500,800,1200)$, up to ten sweeps were performed to converge the sweep energy, defined as the lowest energy out of all the block configurations in each sweep. As noted by Legeza and Fath, ${ }^{22}$ and Chan and Head-Gordon, ${ }^{16}$ there is a linear relationship be-

TABLE I. Orbital orderings. How to read: The numbers go from left to right, top to bottom; thus, at the end of the first row (63), continue to the beginning of the second row (52). The numbers represent the index by increasing Hartree-Fock eigenvalue; the even numbers represent $\alpha$ orbitals, and the odd, $\beta$. Thus, in Hartree-Fock eigenvalue order, the $\alpha$ orbitals are labeled $0,2,4,6 \ldots$, while the $\beta$ orbitals are labeled $1,3,5,7 \ldots$. To obtain the ordering used in this work,

\begin{tabular}{|c|c|c|c|c|c|c|c|c|c|c|c|c|c|c|c|c|c|}
\hline $\begin{array}{l}r_{e} \\
30\end{array}$ & 31 & 40 & 41 & 72 & 73 & 60 & 61 & 50 & 51 & 8 & 9 & 20 & 21 & 28 & 29 & 62 & 63 \\
\hline 52 & 53 & 6 & 7 & 18 & 19 & 48 & 49 & 78 & 79 & 66 & 67 & 36 & 37 & 14 & 15 & 2 & 3 \\
\hline 10 & 11 & 70 & 71 & 38 & 39 & 0 & 1 & 76 & 77 & 44 & 45 & 22 & 23 & 12 & 13 & 54 & 55 \\
\hline 4 & 5 & 16 & 17 & 34 & 35 & 32 & 33 & 64 & 65 & 80 & 81 & 58 & 59 & 68 & 69 & 46 & 47 \\
\hline 24 & 25 & 26 & 27 & 42 & 43 & 74 & 75 & 56 & 57 & & & & & & & & \\
\hline \multicolumn{18}{|l|}{$1.5 r_{e}$} \\
\hline 74 & 77 & 40 & 41 & 26 & 25 & 58 & 59 & 36 & 37 & 66 & 67 & 52 & 53 & 68 & 69 & 56 & 57 \\
\hline 4 & 5 & 12 & 13 & 16 & 17 & 34 & 33 & 24 & 27 & 46 & 47 & 80 & 81 & 30 & 29 & 42 & 43 \\
\hline 76 & 75 & 0 & 1 & 2 & 3 & 10 & 11 & 14 & 15 & 32 & 35 & 60 & 61 & 70 & 71 & 54 & 55 \\
\hline 6 & 9 & 48 & 49 & 62 & 63 & 18 & 21 & 22 & 23 & 44 & 45 & 78 & 79 & 20 & 19 & 8 & 7 \\
\hline 50 & 51 & 64 & 65 & 28 & 31 & 38 & 39 & 72 & 73 & & & & & & & & \\
\hline
\end{tabular}
replace each number below, in order, by the corresponding Hartree-Fock orbital. Orbitals occupied in the Hartree-Fock reference are indicated in bold. 


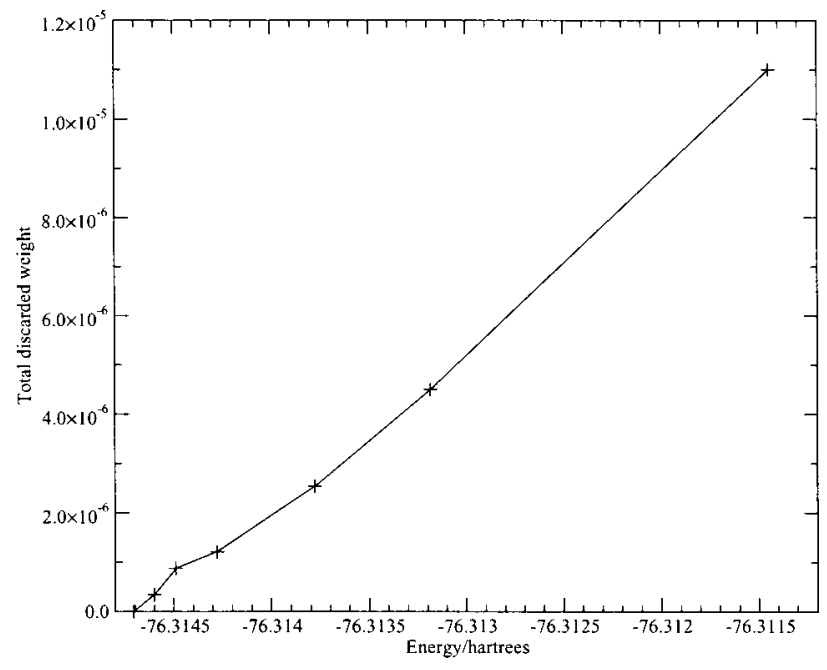

FIG. 2. Total discarded density matrix weight after renormalization against total energy, for $\mathrm{H}_{2} \mathrm{O}$ at $r_{e}$.

tween the magnitude of the total discarded weight in the density matrix, and the error in the total energy. Thus, plotting the sweep energy against the largest total discarded weight per sweep leads to linear plots as shown in Fig. 2. We have used these in extrapolations to provide estimates of the errors in our calculations. The advantage of extrapolating using the discarded weight rather than the recently proposed theoretical convergence behavior $|\delta E| \sim \exp \left(-\kappa(\ln M)^{2}\right)$ (obtained in Ref. 17) is that only a linear fit need be performed for an extrapolation.

For the larger $M$ values (1800-6000) only one or two sweeps at each $M$ value were performed. Thus, during each sweep, the number of states retained in the system block and environment block (built in the previous sweep) are different. This is indicated in Table II by the notation 2400/1800, indicating 2400 many body states in the system and 1800 many body states in the environment. The small number of sweeps does not result in fully converged sweep energies (and thus the DMRG results are not reported to the same number of significant figures as the other entries in Table II), but of course all the results are variational upper bounds to the sweep energies at those $M$ values.

To the best of our knowledge, the largest DMRG calculations on systems with this number of orbitals, have used $M$ values of up to about 1000. (In the work of Mitrushenkov et al., although larger $M$ values appear to be used, the block configuration is different, and thus the effective $M$ values for comparison with this work should be divided by 2 .) Since the cost of our DMRG algorithm is $O\left(M^{3} k^{3}\right)$ time and $O\left(M^{2} k^{3}\right)$ storage (where $k$ is the number of orbitals), our current calculations are two orders of magnitude larger than what has previously been reported, in terms of computational cost and storage. These calculations are now possible because of recent advances we have made to our DMRG algorithm. In particular, we have fully parallelized the DMRG algorithm for distributed memory architectures. In this work, we have run our code on up to 64 processors of the NERSC IBM SP3, which has given us access to 64 IBM Power3/375 CPUs, $256 \mathrm{~Gb}$ of memory, and $1 \mathrm{~Tb}$ of disk. The sweep at
TABLE II. Calculations on $\mathrm{H}_{2} \mathrm{O}$ using the DMRG and traditional quantum chemical methods. Energies in $E_{h}$, errors (from exact value) in $m E_{h}$.

\begin{tabular}{lllll}
\hline \hline & $r_{e}$ & & $1.5 r_{e}$ \\
& Energy & Error & Energy & Error \\
\hline RHF & -76.057621 & 257.094 & -75.817273 & 313.777 \\
UHF & $\mathrm{n} / \mathrm{a}$ & $\mathrm{n} / \mathrm{a}$ & -75.852670 & 278.380 \\
CISD & -76.296239 & 18.476 & -76.092255 & 38.795 \\
CISDT & -76.302586 & 12.129 & -76.103767 & 27.272 \\
CISDTQ & -76.314001 & 0.714 & -76.128604 & 2.446 \\
UCCSD & $\mathrm{n} / \mathrm{a}$ & $\mathrm{n} / \mathrm{a}$ & -76.111899 & 19.151 \\
CCSD & -76.307118 & 7.597 & -76.115089 & 15.961 \\
CCSD(T) & -76.314141 & 0.574 & -76.129442 & 1.608 \\
CCSDT & -76.314294 & 0.421 & -76.129387 & 1.663 \\
CCSDTQ & -76.314696 & 0.019 & -76.130885 & 0.165 \\
& & & & \\
800 & -76.3143 & 0.4 & -76.1302 & 0.9 \\
1200 & -76.3145 & 0.2 & -76.1307 & 0.4 \\
1800/1200 & -76.31458 & 0.13 & -76.13084 & 0.22 \\
2400/1800 & -76.31464 & 0.07 & -76.13095 & 0.11 \\
3200/2400 & -76.31468 & 0.03 & $\mathrm{n} / \mathrm{a}$ & \\
$3600 / 2400$ & $\mathrm{n} / \mathrm{a}$ & $\mathrm{n} / \mathrm{a}$ & -76.13101 & 0.04 \\
$5400 / 3200$ & -76.31471 & 0.005 & $\mathrm{n} / \mathrm{a}$ & \\
$6000 / 3600$ & $\mathrm{n} / \mathrm{a}$ & & -76.13104 & 0.01 \\
$\infty$ & -76.314715 & & -76.131050 & \\
\hline \hline & & & & \\
\hline
\end{tabular}

$M=6000 / 3600$ took over $150 \mathrm{~h}$ of wall time, $250 \mathrm{~Gb}$ of memory and $800 \mathrm{~Gb}$ of disk. We shall present full details of our new algorithm elsewhere.

In Table II we present our DMRG results. Our final computed energies are $-76.314715 \pm 0.000010 E_{h}$ and $-76.131050 \pm 0.000010 E_{h}$ for the $r_{e}$ and $1.5 r_{e}$ geometries respectively. The exact nonrelativistic total energy has been been estimated by several workers, most recently by Luchow et al. ${ }^{23}$ as $-76.438 E_{h}$; the difference between this and our energy is $-0.123 E_{h}$ and is solely attributable to insufficiencies in the one-particle basis. The FCI energies of the $\mathrm{O}$ and $\mathrm{H}$ atoms in this basis are -74.962350 and $-0.499944 E_{h}$ respectively, which yields an atomization energy of $0.352477 E_{h}$, or $221.183 \mathrm{kcal} / \mathrm{mol}$.

At both $r_{e}$ and $1.5 r_{e}$, the convergence of the DMRG calculations with increasing $M$ is quite similar. Based on these results, we see that for systems of this size, using about 800 states is sufficient to reach chemical accuracy $\left(<1 m E_{h}\right)$. This is to be contrasted with the results from CI and $\mathrm{CC}$ theory, which are single-reference theories. At the equilibrium geometry, chemical accuracy is achieved using the standard $\operatorname{CCSD}(\mathrm{T})$ method, which has an error of only $0.574 m E_{h}$, and by the time one includes all connected quadruple excitations in CCSDTQ, the error is reduced to only $0.019 m E_{h}$. However, at the stretched geometry, all the CI and $\mathrm{CC}$ results deteriorate by an order of magnitude. The $\mathrm{CC}$ errors at this geometry must be interpreted with care, as it is now understood that restricted $\mathrm{CC}$ theories yield energies below the variational energy as bonds are stretched (see, e.g., Ref. 24). Similarly, the perturbative triples $(T)$ correction leads the $\operatorname{CCSD}(\mathrm{T})$ result to lie below the full CCSDT result at $1.5 r_{e}$, which is a precursor to the divergence of the perturbative correction at long bond-lengths. The magnitude of the connected quadruples correction at $1.5 r_{e}$ is very large, 
about $1.5 m E_{h}$. The unrestricted CCSD theory also performs significantly worse at this geometry than the corresponding restricted CCSD theory at the equilibrium geometry. All these are indications of the true multiconfigurational character of the wave function at intermediate stretched geometries, which is hard to capture using traditional single-reference theories, but which poses little difficulty for the DMRG. Elsewhere we have emphasized the intrinsically multiconfigurational nature of the DMRG. ${ }^{16}$ We may also understand the DMRG from the viewpoint of valence bond theory. The most important correlations, as a bond is stretched, are those that correlate an occupied bonding orbital with its corresponding virtual antibonding orbital, as emphasized in perfect-pairing valence bond theory. If we generalize the concept of a pair to an orbital cluster of the type shown in Table I, and further allow correlations between different orbital clusters (in order of increasing importance, as $M$ is increased), we recover the DMRG picture of bond dissociation.

In conclusion, we have demonstrated, by computing the exact energy of water in a large basis, that we can now obtain numerically exact solutions for systems considerably larger than can be contemplated using traditional FCI algorithms. This has been possible using a new high-performance DMRG algorithm that we have developed. Studies on a number of challenging chemical systems are currently under way.

We dedicate this work to Professor N. C. Handy, who as always, pointed G. K.-L. C. in the right direction. We are also grateful to Professor R. J. Bartlett and Dr. M. Musial, who very kindly computed the CISDTQ and CCSDTQ numbers reported in this work. The computations were carried out at the NERSC supercomputer center, via a grant from the Department of Energy, Office of Basic Energy Sciences, Sci-
DAC Computational Chemistry Program (Grant No. DEFG02-01ER403301), and the NERSC staff (in particular D. Skinner) are thanked for their assistance in many technical matters.

${ }^{1}$ N. C. Handy, Chem. Phys. Lett. 74, 280 (1980).

${ }^{2}$ P. J. Knowles and N. C. Handy, Chem. Phys. Lett. 111, 315 (1984).

${ }^{3}$ P. J. Knowles, Chem. Phys. Lett. 155, 513 (1989).

${ }^{4}$ J. Olsen, B. O. Roos, P. Jorgensen, and H. J. A. Jensen, J. Chem. Phys. 89, 2185 (1988).

${ }^{5}$ J. Olsen, P. Jorgensen, and J. Simons, Chem. Phys. Lett. 169, 463 (1990).

${ }^{6}$ R. J. Harrison and S. Zarrabian, Chem. Phys. Lett. 158, 393 (1989).

${ }^{7}$ G. L. Bendazzoli and S. Evangelisti, J. Chem. Phys. 98, 3141 (1993).

${ }^{8}$ E. Rossi, G. L. Bendazzoli, S. Evangelisti, and D. Maynau, Chem. Phys. Lett. 310, 530 (1999).

${ }^{9}$ K. G. Wilson, Rev. Mod. Phys. 55, 583 (1983).

${ }^{10}$ S. R. White, Phys. Rev. Lett. 69, 2863 (1992).

${ }^{11}$ S. R. White and R. M. Noack, Phys. Rev. Lett. 68, 3487 (1992), and references therein.

${ }^{12}$ G. Fano, F. Ortolani, and L. Ziosi, J. Chem. Phys. 108, 9246 (1998).

${ }^{13}$ S. R. White and R. L. Martin, J. Chem. Phys. 110, 4127 (1999).

${ }^{14}$ S. Daul, I. Ciofini, C. Daul, and S. R. White, Int. J. Quantum Chem. 79, 331 (2000).

${ }^{15}$ A. O. Mitrushenkov, G. Fano, F. Ortolani, R. Linguerri, and P. Palmieri, J. Chem. Phys. 115, 6815 (2001).

${ }^{16}$ G. K.-L. Chan and M. Head-Gordon, J. Chem. Phys. 116, 4462 (2002).

${ }^{17}$ G. K.-L. Chan, P. W. Ayers, and E. S. Croot, J. Stat. Phys. 109, 289 (2002).

${ }^{18}$ P. Saxe, H. F. Schaefer III, and N. C. Handy, Chem. Phys. Lett. 79, 202 (1981).

${ }^{19}$ C. W. Bauschlicher and P. R. Taylor, J. Chem. Phys. 85, 2779 (1986).

${ }^{20}$ J. Olsen, P. Jorgensen, H. Koch, A. Balkova, and R. J. Bartlett, J. Chem. Phys. 104, 8007 (1995).

${ }^{21}$ P. O. Widmark, P. A. Malmqvist, and B. Roos, Theo. Chim. Acta, 77, 291 (1990).

${ }^{22}$ O. Legeza and G. Fath, Phys. Rev. B 53, 14349 (1996).

${ }^{23}$ A. Luchow, J. B. Anderson, and D. Feller, J. Chem. Phys. 106, 7706 (1997).

${ }^{24}$ S. R. Gwaltney, E. F. C. Byrd, T. V. Voorhis, and M. Head-Gordon, Chem. Phys. Lett. 353, 359 (2002). 